\title{
THE ELECTROMAGNETIC SPECTRUM OF THE CRAB NEBULA
}

\author{
KRISHNA M. V. APPARAO*† \\ Smithsonian Astrophysical Observatory and Harvard College Observatory, \\ Cambridge, Mass., U.S.A.
}

The electromagnetic spectrum of the Crab Nebula has been determined experimentally in the radio, optical, and X-ray regions [1], in which it follows a power law of the type $S(v)=A v^{-\alpha}$, where $S(v)$ is the power (in watts $/ \mathrm{m}^{2} \sec \mathrm{Hz}$ ), $A$ and $\alpha$ are constants, and $v$ is the frequency in $\mathrm{Hz}$. Recent measurements [2-5], however, show a deviation from a power law in the microwave region (see Figure 1). In this paper, we

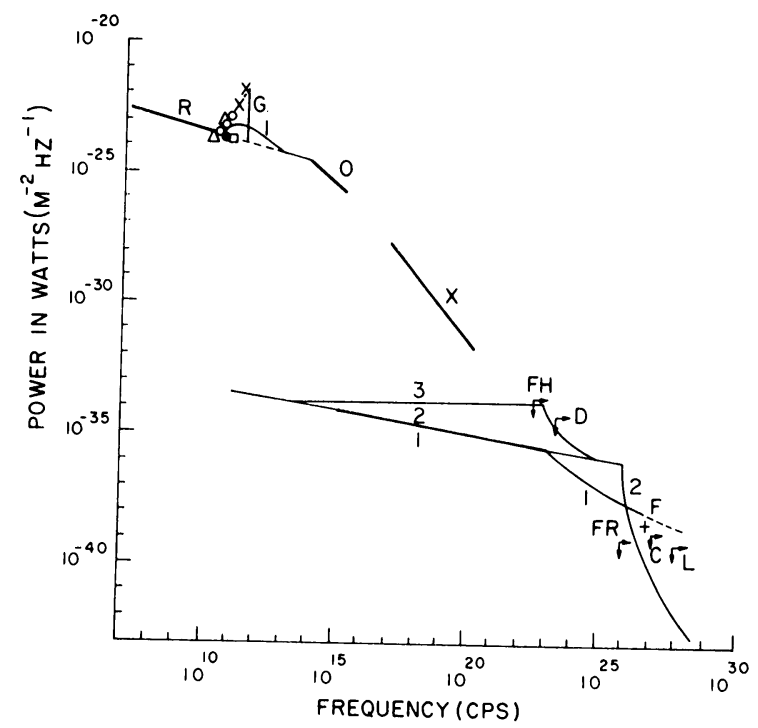

Fig. 1. The electromagnetic spectrum of the Crab Nebula. The thick lines $\mathrm{R}, \mathrm{O}$, and $\mathrm{X}$ are the observed radio, optical, and X-ray spectra. The symbols around $10^{10} \mathrm{~Hz}$ indicate the observations of authors given in references [2-7]. The points with double arrows are upper limits and are integral values as given by Fazio et al. [10], Delvaille et al. [11], Fazio et al. [12], Chudakov et al. [13], Long et al. [14], and Fegan et al. [15]. The various curves are explained in the text. Curve 3 is the present calculation.

investigate the origin of this deviation and calculate the $\gamma$-ray spectrum due to this increase in the microwave photons via the Compton scattering from high-energy electrons.

The radio spectrum between the frequencies $2 \times 10^{7}$ and $2 \times 10^{10} \mathrm{~Hz}$ follows a power law of the form $v^{-\alpha}$, with $\alpha=0.28 \pm 0.05$. Between the frequencies $3 \times 10^{10}$ and

* On leave from the Tata Institute of Fundamental Research, Bombay, India.

+ This work was performed while the author held a U.S. National Academy of Sciences Senior Post-doctoral fellowship supported by the Smithsonian Institution. 
$3 \times 10^{11}$, the new observations indicate $\alpha \simeq-2$. (This change in the exponent is being disputed by Hobbs et al. [6] and Oliver et al. [7].) In the near-infrared and optical regions $\left(v>10^{14} \mathrm{~Hz}\right), \alpha \sim 0.8$. In the $\mathrm{X}$-ray region, $\alpha \simeq 1$.

The difference in the exponent $\alpha$ between the radio spectrum and the optical spectrum is usually understood in terms of a model where there is continuous injection of electrons and where the electrons lose their energy predominantly by synchrotron radiation. Shklovsky [1] suggests that the change in slope at $v \sim 3 \times 10^{10} \mathrm{~Hz}$ could be due to injection of energetic electrons at the time of the explosion of the supernova; again due to synchrotron radiation, the electrons bunch together at a certain energy. It turns out that this hypothesis does not explain the increase of microwave radiation.

Let us consider the solution of the kinetic equation [8] for the differential energy spectrum of electrons $N(E, t)$, with a burst-injection spectrum at the time of the explosion of the form $K E^{-\gamma}$ (K and $\gamma$ are constants) and a continuous injection of the term $q E^{-\gamma_{1}}$ ( $q$ and $\gamma_{1}$ are constants). With only the synchrotron radiation as the main energy-loss process, the solution is

$$
\begin{aligned}
N(E, t) & =K E^{-\gamma}(1-\beta t E)^{\gamma-2} \\
& +\left[q E^{-\left(\gamma_{1}+1\right)} / \beta\left(\gamma_{1}-1\right)\right]\left[1-(1-\beta t E)^{\gamma_{1}-1}\right] .
\end{aligned}
$$

Here $t$ is the age of the nebula, and $\beta$ is a function of the magnetic field $H$ and is given by the equation for synchrotron loss, $(\mathrm{d} E / \mathrm{d} t)=-\beta E^{2}$. Equation (1) leads to the asymptotic forms

$$
N(E, t)= \begin{cases}K E^{-\gamma}+q t E^{-\gamma_{1}} & \text { for } \quad E \ll 1 / \beta t \\ \frac{q E^{-\left(\gamma_{1}+1\right)}}{\beta\left(\gamma_{1}-1\right)} & \text { for } \quad E \gg 1 / \beta t .\end{cases}
$$

Comparing the asymptotic forms of the synchrotron spectra obtained from Equation (2) with the observed optical and radio spectra and using $H=3 \times 10^{-4}$ gauss, we obtain $K, q, \gamma$, and $\gamma_{1}$. Using these, the actual electron spectrum, we derive $N(E, T)$, where $T$ is the present age of the Crab Nebula. The synchrotron spectrum from this $N(E, T)$ is obtained between $10^{10} \mathrm{~Hz}$ and $10^{14} \mathrm{~Hz}$ (plotted as curve 1 in Figure 1). We see that such a burst injection is not sufficient to account for the observations. Beckman et al. [5] suggest that excess radiation in the microwave region may be due to a cool gas. We fit a Planck spectrum to the observations with the maximum at $1.2 \mathrm{~mm}$ (the observation with the highest frequency in the microwave region); the curve is shown as $G$ in Figure 1.

We have calculated the $\gamma$-radiation due to the Compton-synchroton process by using the model given by Gould [9] and bearing in mind that the excess microwave photons are not of synchrotron origin. The $\gamma$-ray spectrum is plotted as curve 3 in Figure 1, along with the predictions from the calculation of Gould (curve 1) and that by Apparao (curve 2), who calculated the $\gamma$-radiation resulting from the Compton scattering of universal microwave photons from the electrons in the Crab Nebula. Selected observations of $\gamma$-radiation are also shown in Figure 1; these are the best upper limits. At energies $E_{\gamma} \geqslant 100 \mathrm{MeV}$, the flux predicted is $2 \times 10^{-5}$ photons $/ \mathrm{cm}^{2}$ 
sec, while the observed limit of Fazio et al. [10] is $3.5 \times 10^{-5}$ photons $/ \mathrm{cm}^{2} \mathrm{sec}$. At energies $E_{\gamma} \geqslant 1 \mathrm{GeV}$, the flux predicted is in $7 \times 10^{-6}$ photons $/ \mathrm{cm}^{2} \mathrm{sec}$, which is to be compared with the limit $1.2 \times 10^{-5}$ photons $/ \mathrm{cm}^{2} \mathrm{sec}$ given by Delvaille et al. [11].

We conclude that the deviation from a power law of the spectrum of the Crab Nebula in the microwave region cannot be accounted for by the synchrotron process under the usual models. It could be of a thermal origin. If the excess of the microwave photons is due to a thermal gas, an upper limit to the temperature of $\sim 5 \mathrm{~K}$ is obtained by comparing the experimental upper limits of $\gamma$-radiation and the predicted fluxes. Further observations of the microwave spectrum and the $\gamma$-ray spectrum in the 100 - to $1000-\mathrm{MeV}$ region will throw light on this question.

\section{References}

[1] For references see Shklovsky, I. S.: 1968, Supernovae, Wiley, London.

[2] Tolbert, C. W.: 1965, Nature 206, 1304.

[3] Tolbert, C. W. and Straiton, A. W.: 1964, Nature 204, 1242.

[4] Kislyakov, A. E. and Na'umov, A. I.: 1968, Soviet Astron.-AJ 11, 6.

[5] Beckman, J. E., Bastin, J. A., and Clegg, P. E.: 1969, Nature 221, 944.

[6] Hobbs, R. W., Corbett, H. H., and Santini, N. J.: 1969, Astrophys. J. Letters 155, L87.

[7] Oliver, J. P., Epstein, E. E., Schorn, R. A., and Soter, S. L.: 1967, Astron. J. 72, 314.

[8] Kardashev, N. S.: 1962, Soviet Astron.-AJ 6, 317.

[9] Gould, R. J.: 1965, Phys. Rev. Lett. 15, 577.

[10] Fazio, G. G., Helmken, H. F., Cavrak, S. J., Jr., and Hearn, D. R.: 1968, Canadian J. Phys. 46, Part 3 (Cosmic Ray Conf. Issue), 427.

[11] Delvaille, J. P., Albats, P., Greisen, K. I., and Ögelman, H. B.: 1968, Canadian J. Phys. 46, Part 3 (Cosmic Ray Conf. Issue), 425.

[12] Fazio, G. G., Helmken, H. F., Rieke, G., and Weekes, T. C.: this volume, p. 192.

[13] Chudakov, A. E., Dadykin, V. L., Zatsepin, V. I., and Nesterova, N. M.: 1964, in Proc. Intern. Conf. Cosmic Rays, Jaipur, India, 4, 199.

[14] Long, C. D., McBreen, B., Porter, N. A., and Weekes, T. C.: 1965, in Proc. Intern. Conf. Cosmic Rays, London, $1,318$.

[15] Fegan, D. J., McBreen, B., O’Mongain, E. P.,JPorter, N. A., and Slevin, P. J.: 1968, Canadian J. Phys. 46, Part 3 (Cosmic Ray Conf. Issue), 433. 